\title{
Una Aproximación Epidemiológica sobre Patologías Orales desde la Perspectiva de la Radiología
}

\author{
An Epidemiological Approach to Oral Pathologies from a Radiology Perspective
}

\author{
Silvia Barrientos Sánchez; Juliana Velosa Porras² \& Adriana Rodríguez Ciódaro³
}

BARRIENTOS, S. S.; VELOSA, P. J. \& RODRÍGUEZ, C. A. Una aproximación epidemiológica sobre patologías orales desde la perspectiva de la radiología. Int. J. Odontostomat., 12(1):43-50, 2018.

RESUMEN: La cavidad de oral es susceptible de alteraciones de diversa índole, que presentan patrones epidemiológicos variables. La caries y la enfermedad periodontal se reconocen como las de mayor prevalencia, pero sobre otras anormalidades que afectan a las estructuras óseas y los dientes se tienen menos datos. El objetivo del trabajo fue determinar la prevalencia de hallazgos radiográficos sugestivos de patología en 10,000 imágenes panorámicas digitales de adultos, obtenidas en diferentes centros de radiología en Bogotá. Se recolectaron radiografías de pacientes mayores de 18 años, de bajo grado de distorsión, con estructuras completas, y adecuado contraste y densidad que permitieran la lectura de las variables a estudiar. La mayoría de las radiografías pertenecen a pacientes de género femenino, y su promedio de edad es de 39 años. El edentulismo parcial es el hallazgo de mayor prevalencia afectando a un $61 \%$ del grupo, seguido de la presencia de tratamientos de endodoncia con un $45 \%$ y en tercer lugar las inclusiones dentales $24,1 \%$. Otros hallazgos corroboran que las lesiones apicales son las lesiones quísticas de mayor prevalencia y un aumento en la rehabilitación con prótesis fija e implantes. Este estudio refleja los efectos de la caries y la enfermedad periodontal que afectan a la población estudiada y su principal consecuencia el edentulismo parcial que afecta a todos los grupos de edad examinados. La endodoncia, sugerente de patología pulpar, también es de alta prevalencia y en gran porcentaje asociada a lesiones apicales, pero en general el estudio sugiere la necesidad de programas de prevención para el adulto, para cumplir con las metas de la Organización Mundial de la Salud con miras a disminuir la perdida de dientes.

PALABRAS CLAVE: Patología oral, epidemiología, radiología.

\section{INTRODUCCIÓN}

La patología oral incluye entidades de origen traumático, inflamatorio, neoplásico, genético e infeccioso, que para ser detectadas, requieren de exámenes complementarios entre los que se cuenta la radiografía panorámica. Esta utiliza tecnología digital con mejor calidad para brindar información más precisa sobre hallazgos tanto dentales como óseos. (American Dental Association Council on Scientific Affairs, 2006) y se reconoce que su sensibilidad y especificidad varían de acuerdo a cada hallazgo, pero es evidente que es un examen valido en estudios epidemiológicos, por ser de bajo costo y seguro (Hugoson et al., 2005; Choi et al., 2011). Con muestras importantes de radiografías se ha podido detectar la prevalencia de dientes incluidos, asimetrías mandibulares, anomalías dentales tanto de número (Fardi et al., 2011) como de forma (Shokri et al., 2014), variaciones anatómicas, quistes y tumores de los maxilares, y alteraciones de crecimiento y desarrollo (Bernaerts et al., 2006; Espinal Botero et al., 2009; Schmuckli et al., 2010). Su utilidad en la identificación postmortem y determinación de la edad de un individuo así como patologías sistémicas que se asocian con hallazgos en las estructuras anatómicas maxilofaciales, como ateromas carotideos (Ngamsom et al., 2015), calcificaciones pulpares asociadas a eventos cardiovasculares (Khojastepour et al., 2013) y cambios en el índice mandibular en pacientes con osteoporosis (Savic Pavicin et al., 2014)

\footnotetext{
${ }^{1}$ Odontóloga, Estomatóloga, MsC Microbiología, Profesor Asociado, ClO, Pontificia Universidad Javeriana, Colombia.

${ }^{2}$ Odontóloga, MsC. Epidemiología, aspirante a PhD, Profesor Asociado, CIO, Pontificia Universidad Javeriana, Colombia.

${ }^{3}$ Bacterióloga, MsC. Microbiología, Profesor Asociado, CIO, Pontificia Universidad Javeriana, Colombia.
} 
son algunos de los ejemplos de la utilidad de la radiografía panorámica.

En Colombia los datos sobre salud oral se han obtenido con estudios de corte clínico, muy útiles para el diagnóstico de caries y enfermedad periodontal, por lo que poco se conoce sobre el comportamiento de otras patologías que afectan a la cavidad oral en este medio. (Ministerio de Salud, 2014) En algunos acercamientos al estudio sobre hallazgos radiográficos en población colombiana, en 228 radiografías se muestran frecuencias de hallazgos del $96 \%$ con afección especialmente del seno maxilar y anomalías de posición y forma de los dientes (Aguilar et al., 2009). En 328 radiografías panorámicas de pacientes entre los 4 y 11 años, se caracterizó la frecuencia de hipodoncia, dilaceración, erupción ectópica, hiperdoncia y microdoncia (García Salazar \& Manotas Arevalo, 2006).

Teniendo en cuenta la evidente utilidad de la radiografía panorámica como medio diagnóstico, y la necesidad de complementar datos epidemiológicos de diferentes patologías orales, se buscó, en 10.000 imágenes panorámicas digitales, de individuos adultos, determinar la frecuencia de hallazgos compatibles con alteraciones dentales y óseas, como aporte al estudio de la salud oral en esta población y que permiten ver el panorama de necesidad de tratamiento odontológico.

\section{MATERIAL Y MÉTODO}

Utilizando un estudio observacional descriptivo aprobado por el Comité de Investigación y Ética de la Facultad de Odontología de la Pontificia Universidad Javeriana y obtenidos los permisos de los distintos Centros de Radiología, se analizaron diez mil (10.000) radiografías panorámicas digitales. La muestra fue escogida por conveniencia, de pacientes mayores de 18 años. Incluyó imágenes digitales con densidad y contraste adecuados para su lectura, que permitieran la visualización completa de las estructuras con la menor distorsión posible. Se calibraron los observadores y se definieron los términos de lectura radiográfica a través de un mapeo sistemático y ordenado por zonas así 1: Seno maxilar derecho, 2: Región nasal, 3: Seno maxilar izquierdo, 4: Cuadrante dentoalveolar superior derecho, 5: Cuadrante dentoalveolar superior izquierdo, 6: Cóndilo mandibular derecho, 7: Escotadura sigmoidea dere- cha, 8: Apófisis coronoides derecha, 9: Rama mandibular derecha, 10: Cuerpo mandibular derecho, 11: Sínfisis mandibular, 12: Cuerpo mandibular izquierdo, 13: Rama mandibular izquierda, 14: Apófisis coronoides izquierda, 15: Escotadura sigmoidea izquierda, 16: Cóndilo mandibular izquierdo, 17: Cuadrante dentoalveolar inferior derecho, 18: Cuadrante dentoalveolar inferior izquierdo, como se ilustra en la Figura 1.

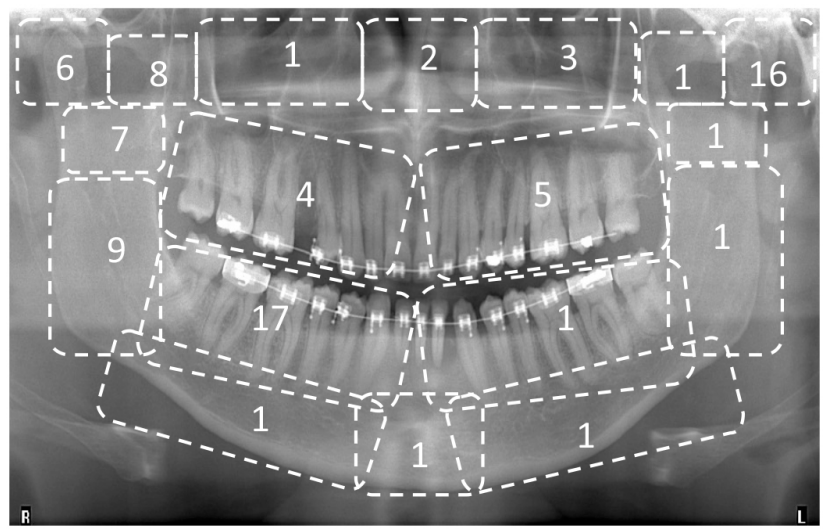

Fig. 1. Mapeo por zonas de la radiografia panorámica para la evaluación

En las estructuras dentales se buscaron hallazgos compatibles con dientes supernumerarios, incluidos, temporales, lesiones apicales, endodoncias, edentulismo total y parcial, prótesis fija, implantes, aparatología ortodontica y restos radiculares, mientras que en hallazgos óseos se registraron aquellos relacionados por su apariencia radiográfica con quistes, tumores y otras lesiones óseas, reabsorción ósea y material de osteosíntesis. Para el análisis de la información los resultados se distribuyeron por género y grupos de edad. Se consideró como hallazgo la presencia de por lo menos un evento, sin tener en cuenta el número de dientes o estructuras afectadas por el mismo hallazgo.

Los datos se registraron en una hoja de Excel y se realizó estadística descriptiva por medio de frecuencias, promedios, desviaciones estándar. Se calcularon pruebas de Chi2 y Kruskal-Wallis y como Post hoc la prueba de Tukey. Se consideró significativo un valor $p \leq 0,05$. Se obtuvieron los OR para establecer asociaciones entre hallazgos que por su naturaleza se pudieran encontrar relacionados, como dientes incluidos con tratamiento ortodóntico y lesiones apicales con tratamiento de endodoncia. 


\section{RESULTADOS}

De las 10.000 radiografías panorámicas analizadas, el $57,8 \%$ pertenecían a mujeres, con una edad promedio de 38,4 (D.E. 15,4) años, mientras en los hombres el promedio fue de 39 años (D.E. 15,6) para un promedio general de 39,2 años (D.E. 15,5).

Del total de las imágenes, 680 radiografías no presentaron algún tipo de hallazgo. El 56,2\% correspondían a mujeres y el 43,8 \% a hombres. El 61,8\% de estas se encontraban en el grupo de 18-28 años seguido del $31,8 \%$ que correspondía a las edades entre 29-38 años. Este 6,8 \% no se asume como sano, debido a que puede tener hallazgos no detectables por este método.

En $9.320(93,2 \%)$ radiografías se encontró un total de 28.561 hallazgos, de los cuales 22.547 correspondían a hallazgos dentales y 6.014 a hallazgos óseos. Teniendo en cuenta que la unidad de análisis fueron las radiografías, se reporta el número de radiografías que presentaban algún tipo de hallazgo. Los de mayor prevalencia fueron el edentulismo parcial, dientes con endodoncia, prótesis fija, diente(s) incluido(s) y reabsorción ósea (Tabla I) (Fig. 2).
El análisis por sexo mostró que las mujeres presentan más edentulismo $(p=0.000)$, mientras que los dientes supernumerarios, lesión (es) apical (es) y restos radiculares son ítems que se presentan con mayor frecuencia en los hombres. En los otros hallazgos el comportamiento por género no muestra diferencia estadísticamente significativa. (Tabla II).

Tabla I. Prevalencia de hallazgos radiográficos encontrados en la población analizada.

\begin{tabular}{lrr}
\hline Característica & $\mathrm{N}$ & \multicolumn{1}{c}{$\%$} \\
\hline Radiografías sin hallazgos & 680 & 6,8 \\
Hallazgos Dentales & & \\
Diente Supernumerario & 128 & 1, \\
Diente Incluido & 2413 & 24,1 \\
Lesión Apical & 1792 & 17,9 \\
Diente Temporal & 66 & 0, \\
Edentulismo Total & 112 & 1, \\
Edentulismo Parcial & 6095 & 60,9 \\
Prótesis Fija & 2766 & 27,6 \\
Implante & 526 & 5, \\
Ortodoncia & 1632 & 16,3 \\
Resto Radicular & 1392 & 13,9 \\
Diente con Endodoncia & 4501 & 45,0 \\
Hallazgos óseos & & \\
Lesión ósea / Quiste o Tumor & 538 & 5, \\
Reabsorción Ósea & 2331 & 23,3 \\
Material de Osteosíntesis & 177 & 1, \\
\hline
\end{tabular}
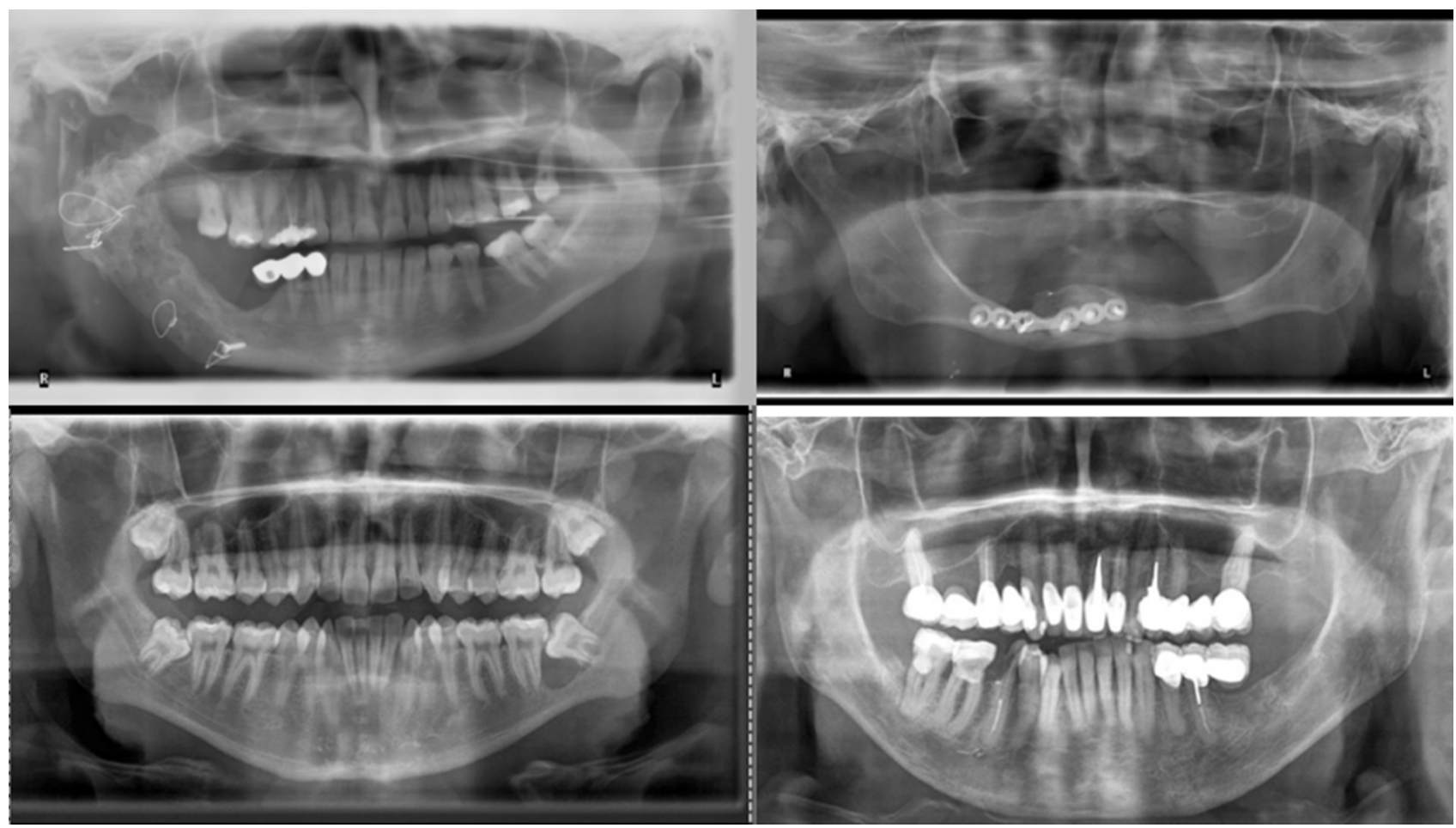

Fig. 2. Radiografías panorámicas digitales con hallazgos radiográficos óseos y dentales. 
BARRIENTOS, S. S.; VELOSA, P. J. \& RODRÍGUEZ, C. A. Una aproximación epidemiológica sobre patologías orales desde la perspectiva de la radiología. Int. J. Odontostomat., 12(1):43-50, 2018.

Tabla II. Distribución de hallazgos radiográficos por sexo.

\begin{tabular}{lccr}
\hline Característica & Femenino & Masculino & Valor p \\
\hline Hallazg os Dentales & 5402 & 3918 & \\
Diente Supernumerario & $61(1,12 \%)$ & $67(1,71 \%)$ & 0,019 \\
Diente Incluido & $1368(25,2 \%)$ & $1045(26,6 \%)$ & 0,14 \\
Lesión Apical & $950(17,5 \%)$ & $842(21,5 \%)$ & 0,000 \\
Diente Temporal & $39(0,72 \%)$ & $27(0,68 \%)$ & 0,90 \\
Edentulismo Total & $74(1,36 \%)$ & $38(0,96 \%)$ & 0,08 ‘ \\
Edentulismo Parcial & $3622(67 \%)$ & $2473(63,1 \%)$ & 0,000 \\
Prótesis Fija & $1634(30,2 \%)$ & $1132(28,8 \%)$ & 0,16 \\
Implante & $294(5,44 \%)$ & $232(5,92 \%)$ & $0,34($ \\
Ortodoncia & $964(17,8 \%)$ & $668(17,04 \%)$ & $0,32($ \\
Resto Radicular & $745(13,7 \%)$ & $647(16,5 \%)$ & 0,000 \\
Diente con Endodoncia & $2622(48,5 \%)$ & $1879(47,9 \%)$ & $0,58 !$ \\
Hallazg os óseos & & & \\
Lesión Ósea / Quiste / Tumor & $304(5,6 \%)$ & $234(5,9 \%)$ & $0,50($ \\
Reabsorción Ósea & $1353(25 \%)$ & $978(24,9 \%)$ & 0,94 ؛ \\
Material de Osteosíntesis & $107(1,98 \%)$ & $70(1,78 \%)$ & $0,53 !$ \\
\hline
\end{tabular}

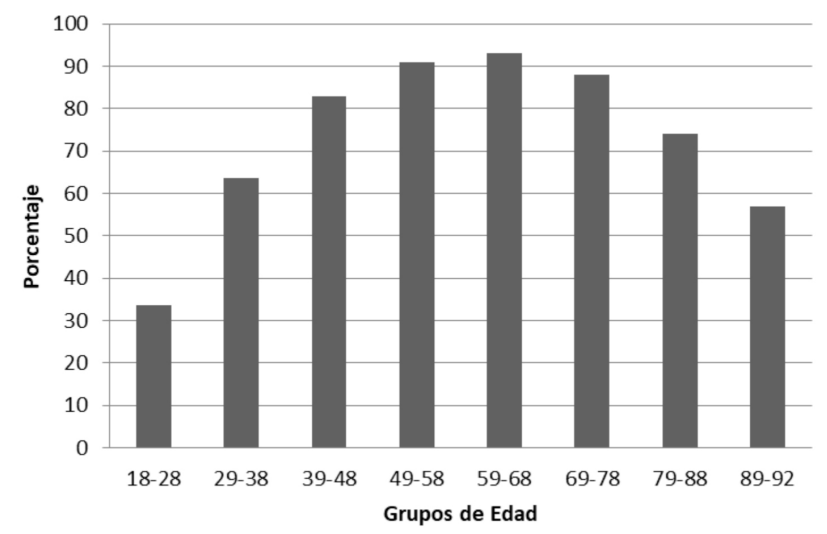

Fig. 3. Prevalencia de edentulismo parcial por grupos de edad.

La edad se dividió en 8 categorías siendo el rango de 18-28 años $(33,7 \%)$ la más frecuente seguida de $29-38$ años $(23,4 \%)$. En cuanto a los hallazgos dentales se encontraron diferencias significativas de acuerdo con la edad en la mayoría de características evaluadas. Con respecto a los hallazgos óseos se encontró que hay diferencias significativas por edad en las lesiones óseas/ quiste o tumor, siendo estas más frecuentes en el grupo de 18 a 28 años de edad. La reabsorción ósea fue más frecuente en el grupo de 49 a 58 seguido de 59-68 (Tabla III).

El análisis post-hoc mostró diferencias significativas en la mayoría de hallazgos por grupo de edad. En la Figura 3 se resaltan los resultados encontrados para el edentulismo parcial, en la que se observa un aumento gradual y proporcional con la edad, hasta cuando es reemplazado por el edentulismo total en la población mayor de 69 años (Tabla III, Fig. 3).

Al realizar el análisis en busca de asociaciones entre hallazgos clinicamente relacionados, se encontró que los dientes con endondoncia se asocian con lesiones apicales $(p=0,000)$ con un OR=3,01 IC $95 \%$ $(2,69-3,36)$ (Fig. 4).

De igual forma se encontró asociación entre la ortodoncia y presentar por lo menos un diente incluido $(p=0,000)$. El riesgo encontrado fue $O R=1,319$ IC 95 $\%(1,17-1,48)$ (Fig. 5).

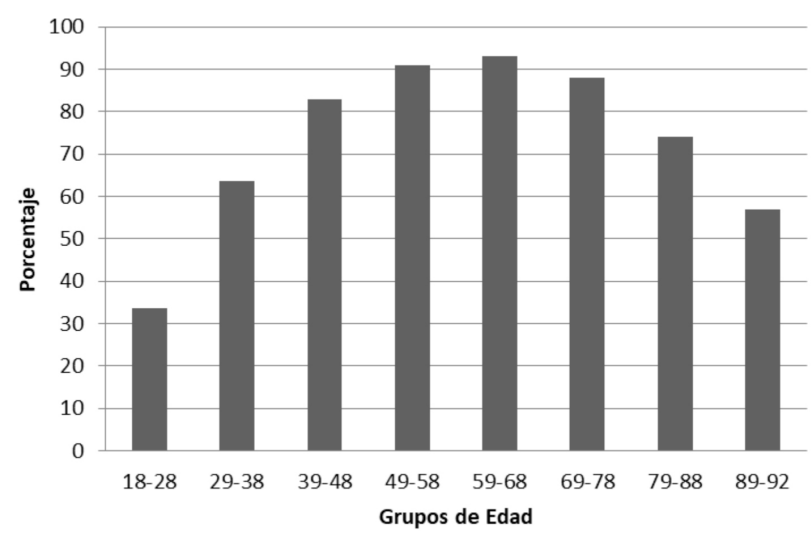

Fig. 4. Asociación entre las variables diente con endodoncia y lesión apical. 
BARRIENTOS, S. S.; VELOSA, P. J. \& RODRÍGUEZ, C. A. Una aproximación epidemiológica sobre patologías orales desde la perspectiva de la radiología. Int. J. Odontostomat., 12(1):43-50, 2018.

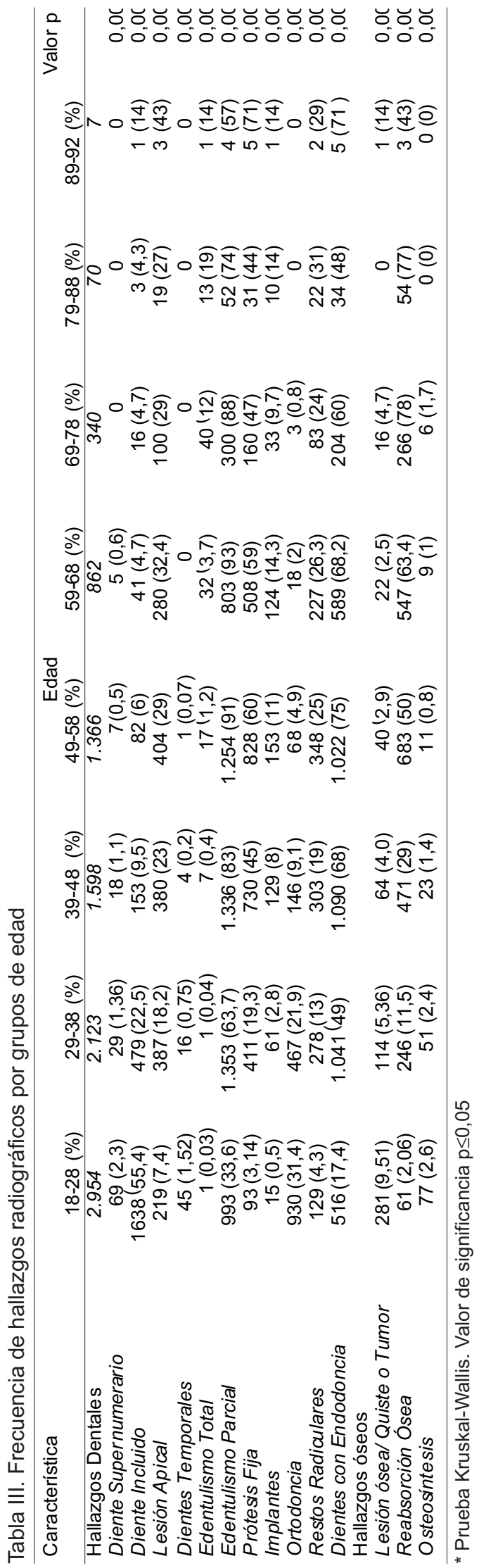

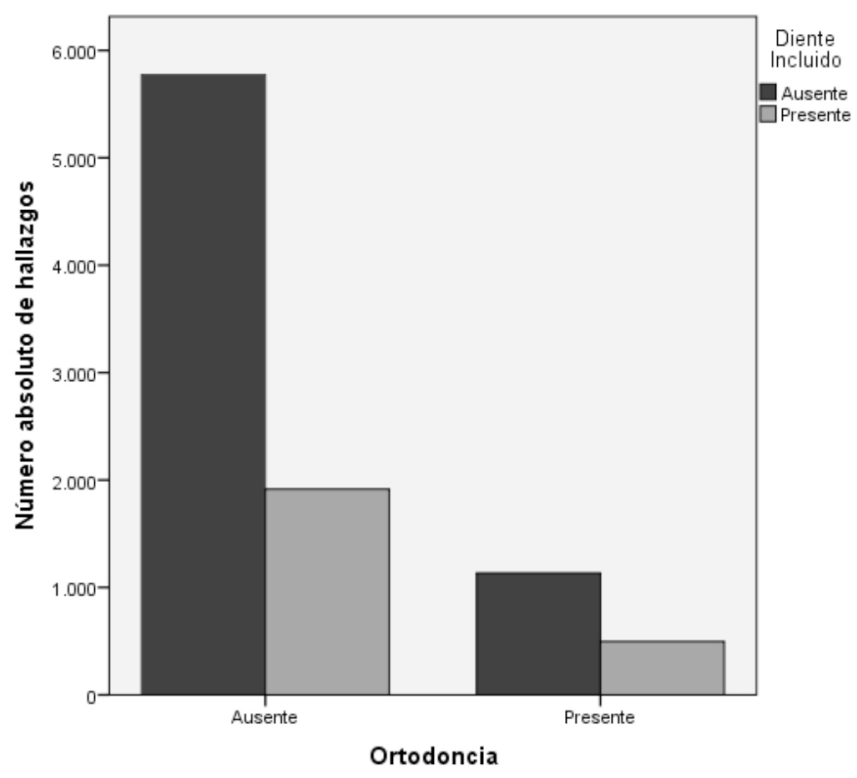

Fig. 5. Asociación entre ortodoncia y diente incluido.

\section{DISCUSIÓN}

El reconocimiento de los perfiles epidemiológicos en una población es de vital importancia para la toma de decisiones en salud pública. Las patologías orales afectan a 3,9 billones de personas y los años de vida asociados con enfermedad oral aumentaron en $20,8 \%$ entre 1990 y 2010 , siendo la caries de dientes permanentes la enfermedad de mayor prevalencia en la carga global de morbilidad (Marcenes et al., 2013). En Colombia el peso de la enfermedad en cavidad oral es de alto impacto debido al poco control de la caries y la enfermedad periodontal y sus secuelas en todos los grupos de edad.

El hallazgo de mayor significancia, es el edentulismo parcial con un $61 \%$ de la población estudiada afectando todos los rangos de edad. EI ENSAB IV en 2014, reportaba que el 51,9\% de las personas entre 15 y 44 años mantenían su dentadura completa en el maxilar superior y $46,7 \%$ en el maxilar inferior con una necesidad de prótesis del $25,8 \%$ para el superior y $7 \%$ para el inferior y $22,3 \%$ necesitaban prótesis porque no la usan y 8,4 y $9,5 \%$ usaban prótesis fija (Ministerio de Salud). Muchos factores influyen en el edentulismo e incluyen la edad, el género, las condiciones sistémicas, también la educación y el acceso al servicio de salud oral. Las mujeres están afectadas de manera significativa en mayor proporción, lo que podría deberse a que encuentran en la exodoncia una solución rápida y de bajo costo. Asimismo el $33 \%$ de la población adulta joven entre 18 a 28 años, habrá perdido por lo menos un diente, diferente de los terceros molares, esto probablemente más por exodoncia de 
premolar es para ortodoncia o trauma. Esta condición casi se duplica en la década de los 28 a 38 años, pasando de 33 a $63 \%$ de individuos edéntulos, es decir el cuidado de la salud oral se reduce sustancialmente en este grupo y probablemente la exodoncia sigue siendo un método simple y económico para los pacientes como ya se dijo y su causa esta mayormente relacionada con caries y enfermedad periodontal. En Bogotá, Colombia en el 2.007, la Secretaria de Salud encuentra que al entrar a la edad adulta se habrán perdido 1,3 dientes, subiendo esta proporción drásticamente a 5,5 dientes entre 29 y 44 años hasta llegar a 17 dientes a los 60 años, datos que muestran que el edentulismo no solo se incrementa en número de afectados sino en la severidad, confirmando la incapacidad del adulto para mantener su salud oral (Alcaldía Mayor de Bogotá D. C., 2011). En una revisión sistemática de la literatura, Kassebaum et al. (2014), señalan como entre 1.990 y 2.010 la tasa de incidencia de edentulismo severo (menos de 9 dientes en boca) a nivel mundial, disminuyó de 374 casos por cada 100.000 habitantes a 205 casos por cada 100.000 habitantes en 2.010 , aunque el incremento de la severidad aumenta con la edad, sin que el autor encuentre diferencias por género. Los datos de este estudio indican que esta población supera ampliamente, con 112 casos de 10.000, solo con edéntulos totales, a la tendencia mundial, a pesar que los mismos autores afirman que Colombia está entre los países con disminución en las tasas de edentulismo (Kassebaum et al.).

El problema del edentulismo es su impacto en la calidad de vida de la persona debido a que trae como consecuencia dificultades funcionales para hablar, masticar, así como dolor, incomodidad estética y limitaciones sociales aún si se utilizan prótesis (De Marchi et al., 2012; Visscher et al., 2014). Adicionalmente la reabsorción ósea consecuente obliga a realizar aumento de reborde para obtener resultados estéticos y funcionales de mayor costo al momento de rehabilitar a un paciente. También es de anotar la necesidad de exodoncia por presencia de restos radiculares $(14,9 \%)$, lo que aumentaría la frecuencia y severidad del edentulismo en esta muestra.

Es interesante encontrar que el $27,7 \%$ de los pacientes edéntulos tienen una prótesis fija, un aumento sustancial desde el ENSAB IV (Ministerio de Salud) y el número de implantes asciende a 5,3\%, dato que indica que aún no es un tratamiento accesible para la población. Con este estudio no es posible determinar la rehabilitación con prótesis removibles, pero se puede pensar que hay más edéntulos pero también con más acceso a la rehabilitación.

El segundo dato de mayor frecuencia es el de la presencia de una o más endodoncias (45\%), siendo este tipo de tratamiento, un indicador de patología pulpar por efecto de la caries generalmente. Al llegar a la edad adulta, $15 \%$ de las personas ya tendrán por lo menos un tratamiento endodóntico, cifra que aumenta dramáticamente en el segundo grupo de 29 a 38 años, donde el $44,5 \%$ ya habrán requerido por lo menos una endodoncia siendo las mujeres quienes tienen más tratamientos de endodoncia. El 19,2\% de los dientes tratados tienen lesiones apicales asociadas de manera estadísticamente significativa aunque este estudio no permite saber la relación causa efecto entre estas dos variables, a pesar de que se detecta una tendencia a que las endodoncias estén subobturadas coincidiendo con otros estudios de población similar, con un OR de 3,01 (Moreno et al., 2013).

La presencia de uno o más dientes incluidos se encontró como el tercer hallazgo más importante en el $24,1 \%$; en este caso los hombres son los más afectados, y con mayor frecuencia los terceros molares. Diferente a estos resultados, en 207 pacientes turcos, se encontró una prevalencia de terceros molares incluidos del 51 \% (Topkara \& Sari, 2013). En un estudio en radiografías panorámicas de 1.020 iraníes, se reporta $57,4 \%$ de personas con dientes incluidos con mayor frecuencia en el género masculino (Hashemipour et al., 2013). Sobre 1.239 radiografías panorámicas en población griega hay $13,7 \%$ de dientes incluidos siendo el de mayor prevalencia el canino, seguido de premolares, dientes supernumerarios y molares (Fardi et al.) Estas diferencias tan marcadas entre distintos grupos poblacionales, podrían deberse a diversidad en los patrones de crecimiento y desarrollo y en que pueden estar ausentes porque han sido extraídos por tratamiento de ortodoncia en edades más tempranas $\mathrm{o}$, menos común, por patologías en los dientes vecinos.

En cuanto a la presencia de aparatología de ortodoncia, como indicador de maloclusión, el porcentaje es del $16,3 \%$ lo que significa que las anomalías de posición dental son también un problema importante en esta población, más aún si se tiene en cuenta que sólo se están contando los casos que están en tratamiento. EI ENSAB IV describe un $33.6 \%$ de población con algún tipo de maloclusión (Ministerio de Salud) mientras que Thilander et al. (2001) encontró que en una población bogotana entre 5 y 17 años, el 
$88 \%$ requerían algún tipo de corrección por ortodoncia. La prevención y el tratamiento de las maloclusiones dentales y esqueléticas, los hace el ortodoncista, que por costos es de difícil acceso para las poblaciones de menores recursos. Un dato interesante del presente estudio, es el hecho de que los pacientes con ortodoncia tienen un riesgo mayor de tener dientes incluidos, probablemente porque la mal oclusión tiene componentes esqueléticos de tamaño, que favorecen la impactación de algunos dientes, en su mayoría terceros molares; es así como el cálculo del OR muestra una relación entre la presencia de ortodoncia y dientes incluidos de 1,319 es decir tener algún tipo de maloclusión aumenta el riesgo para que ciertos dientes se queden retenidos.

La prevalencia de lesiones compatibles con quistes, tumores o patología ósea es baja (5,8 \%) y los casos se relacionan más con lesiones reactivas como la osteítis condensante, con muy pocos quistes odontogénicos, teniendo en cuenta solamente parámetros radiográficos (Raitz et al., 2009). En un estudio sobre 12.197 pacientes a lo largo de 20 años, se encontró una frecuencia de 1.310 lesiones en su gran mayoría quistes radiculares, dato similar al presente trabajo. (Tortorici et al., 2008) En India se reporta en una muestra de 2.075 biopsias, una incidencia del $5,78 \%$ de tumores odontogénicos clasificados como ameloblastomas, queratoquistes, odontomas, tumor odontogénico adenomatoide con una mayor predilección por el hueso mandibular (Varkhede et al., 2011).

Otros datos obtenidos de esta muestra evidencian una prevalencia de $1,9 \%$ de material de osteosíntesis asociado a trauma maxilofacial o a cirugía ortognática con una tendencia a que se encuentre más material de osteosíntesis compatible con corrección de anomalías esqueléticas probablemente por el auge de la cirugía estética. En México la prevalencia de cirugía maxilofacial por trauma es del $9,4 \%$ en un servicio especializado sobre 1611 pacientes en 5 años (Medina-Solis et al., 2004) y en cuanto a anomalías dentomaxilofaciales con indicación quirúrgica se halló un $39 \%$, en un grupo similar (Delgado Galíndez \& Villalpaondo Carreón, 2005).

La Organización Mundial de la Salud se propuso para el año 2.000 una reducción a menos del $50 \%$ del edentulismo en los pacientes entre 35 y 48 años y $5 \%$ en mayores de 65 años (World Health Organization, 2018). Esto implicaba un reto en el control de la caries desde la infancia y la enfermedad periodontal en la edad adulta. Los resultados de esta investigación muestran que los efectos de estas dos patologías siguen siendo de muy alto impacto teniendo en cuenta que el edentulismo consecuente es un indicador fundamental de salud oral (Reddy et al., 2012) y refleja, en primer lugar el poco acceso a la prevención, educación y a los servicios de salud oral en este caso de la población adulta (Ministerio de Salud).

BARRIENTOS, S. S.; VELOSA, P. J. \& RODRÍGUEZ, C. A. An epidemiological approach to oral pathologies from a radiology perspective. Int. J. Odontostomat., 12(1):43-50, 2018.

ABSTRACT: The oral cavity is susceptible to alterations of diverse nature that present variable epidemiological patterns. Caries and periodontal disease are recognized as having the highest prevalence, but thereare less data on other abnormalities that affect bone structures and teeth. The objective of the study was to determine the prevalence of radiographic findings suggestive of pathology in 10,000 digital panoramic images of adults, obtained at different radiology centers in Bogotá. Radiographs were collected from patients over 18 years of age, with a low degree of distortion, with complete structures, and adequate contrast and density to allow the reading of the variables to be studied. Most of the radiographs belong to female patients, and their average age is 39 years. Partial edentulism is the most prevalent finding affecting $61 \%$ of the group, followed by the presence of endodontic treatments with $45 \%$ and thirdly the dental inclusions $24.1 \%$. Other findings corroborate that the apical lesions are the cystic lesions with a higher prevalence and an increase in rehabilitation with fixed prostheses and implants. This study reflects the effects of caries and periodontal disease that affect the population studied and its main consequence is the partial edentulism that affects all age groups examined. Endodontics, suggestive of pulpal pathology, is also of high prevalence and a large percentage associated with apical lesions, but in general the study suggests the need for adult prevention programs to meet the goals of the World Health Organization with a view to reducing tooth loss. radiology.

KEY WORDS: Ora pathology, epidemiology,

\section{REFERENCIAS BIBLIOGRÁFICAS}

Aguilar, O.; Gallego Gómez, C. L.; Iriarte Agudelo, A. M. \& Quintero, I. S. Prevalencia de hallazgos en radiografías panorámicas de rutina. Rev. Nac. Odontol. U. C. C., 5(9):15-20, 2009.

Alcaldía Mayor de Bogotá D. C. Salud Oral. Bogotá, Secretaría de Salud de Bogotá, 2011.

American Dental Association Council on Scientific Affairs. The use of dental radiographs: update and recommendations. J. Am. Dent. Assoc., 137(9):1304-12, 2006. 
Bernaerts, A.; Vanhoenacker, F. M.; Hintjens, J.; Chapelle, K.; Salgado, R.; De Foer, B. \& De Schepper, A. M. Tumors and tumor-like lesions of the jaw mixed and radiopaque lesions. JBRBTR, 89(2):91-9, 2006.

Choi, J. W. Assessment of panoramic radiography as a national oral examination tool: review of the literature. Imaging Sci. Dent., 41(1):1-6, 2011.

De Marchi, R. J.; Hilgert, J. B.; Hugo, F. N.; Santos, C. M.; Martins, A. B. \& Padilha, D. M. Four-year incidence and predictors of tooth loss among older adults in a southern Brazilian city. Community Dent. Oral Epidemiol., 40(5):396-405, 2012.

Delgado Galíndez, B. \& Villalpaondo Carreón, M. Incidence of dentofacial deformities in the Specialty Hospital of the Instituto Mexicano del Seguro Social. Rev. Med. Inst. Mex. Seguro Soc., 43(2):155-9, 2005.

Espinal Botero, G.; Manco Guzmán, H. A.; Aguilar Méndez, G.; Castrillón Pino, L.; Rendón Giraldo, J. E. \& Marín Botero, M. L. Estudio retrospectivo de anomalías dentales y alteraciones óseas de maxilares en niños 5 a 14 años de las Clínicas de la Facultad de Odontología de la Universidad de Antioquia. Rev. Fac. Odontol. Univ. Antioq., 21(1):50-64, 2009.

Fardi, A.; Kondylidou-Sidira, A.; Bachour, Z.; Parisis, N. \& Tsirlis, A. Incidence of impacted and supernumerary teeth-a radiographic study in a North Greek population. Med. Oral Patol. Oral Cir. Bucal, 16(1):e56-61, 2011.

García Salazar, C. \& Manotas Arevalo, I. Hallazgos imagenológicos en radiografía panorámica, de alteraciones en el crecimiento y desarrollo dentario en niños entre 4 y 11 años de edad, atendidos en una clínica odontológica, durante el período 2001- 2004. Duazary, 3(1):45-54, 2006.

Hashemipour, M. A.; Tahmasbi-Arashlow, M. \& Fahimi-Hanzaei, F. Incidence of impacted mandibular and maxillary third molars: a radiographic study in a Southeast Iran population. Med. Oral Patol. Oral Cir. Bucal, 18(1):e140-5, 2013.

Hugoson, A.; Koch, G.; Göthberg, C.; Helkimo, A. N.; Lundin, S. A.; Norderyd, O.; Sjödin, B. \& Sondell, K. Oral health of individuals aged 3-80 years in Jönköping, Sweden during 30 years (19732003). II. Review of clinical and radiographic findings. Swed. Dent. J., 29(4):139-55, 2005.

Kassebaum, N. J.; Bernabé, E.; Dahiya, M.; Bhandari, B.; Murray, C. J. \& Marcenes, W. Global burden of severe tooth loss: A systematic review and meta-analysis. J. Dent. Res., 93(7 Suppl.):20S-28S, 2014

Khojastepour, L.; Bronoosh, P.; Khosropanah, S. \& Rahimi, E. Can dental pulp calcification predict the risk of ischemic cardiovascular disease? J. Dent. (Tehran), 10(5):456-60, 2013.

Marcenes, W.; Kassebaum, N. J.; Bernabé, E.; Flaxman, A.; Naghavi, M.; Lopez, A. \& Murray, C. J. Global burden of oral conditions in 1990-2010: a systematic analysis. J. Dent. Res., 92(7):592-7, 2013.

Medina-Solis, C. E.; Córdova-González, J. L.; Casanova-Rosado, A. J. \& Zazueta-Hernández, M. A. Maxillofacial fractures and associated factors in Campeche Mexico, Instituto Mexicano del Seguro Social medical insurance policyholders. Retrospective analysis 1994-1999. Gac. Med. Mex., 140(1):27-32, 2004.

Ministerio de Salud. IV Estudio Nacional de Salud Bucal ENSAB IV. Situación en Salud Bucal. Para Saber Cómo Estamos y Saber Qué Hacemos. Bogotá, Ministerio de Salud, República de Colombia, 2014.

Moreno, J. O.; Alves, F. R.; Gonçalves, L. S.; Martinez, A. M.; Rôças, I. N. \& Siqueira, J. F. Jr. Periradicular status and quality of root canal fillings and coronal restorations in an urban Colombian population. J. Endod., 39(5):600-4, 2013.

Ngamsom, S.; Arayasantiparb, R.; Pornprasertsuk-Damrongsri, S. \& Sureephong, B. Relationship between calcified carotid atheromas in digital panoramic radiographs and underlying systemic diseases in implant patients. J. Investig. Clin. Dent., 6(4):301-6, 2015.

Raitz, R.; Assunção Júnior, J. N.; Correa, L. \& Fenyo-Pereira, M. Parameters in panoramic radiography for differentiation of radiolucent lesions. J. Appl. Oral Sci., 17(5):381-7, 2009.

Reddy, N. S.; Reddy, N. A.; Narendra, R. \& Reddy, S. D. Epidemiological survey on edentulousness. J. Contemp. Dent. Pract., 13(4):562-70, 2012.

Savic Pavicin, I.; Dumancic, J.; Jukic, T.; Badel, T. \& Badanjak, A. Digital orthopantomograms in osteoporosis detection: mandibular density and mandibular radiographic indices as skeletal BMD predictors. Dentomaxillofac. Radiol., 43(7):20130366, 2014.

Schmuckli, R.; Lipowsky, C. \& Peltomäki, T. Prevalence and morphology of supernumerary teeth in the population of a Swiss community. Short communication. Schweiz. Monatsschr. Zahnmed., 120(1):987-93, 2010.

Shokri, A.; Poorolajal, J.; Khajeh, S.; Faramarzi, F. \& Kahnamoui, H. $\mathrm{M}$. Prevalence of dental anomalies among 7- to 35-year-old people in Hamadan, Iran in 2012-2013 as observed using panoramic radiographs. Imaging Sci. Dent., 44(1):7-13, 2014.

Thilander, B.; Pena, L.; Infante, C.; Parada, S. S. \& de Mayorga, C. Prevalence of malocclusion and orthodontic treatment need in children and adolescents in Bogota, Colombia. An epidemiological study related to different stages of dental development. Eur. J. Orthod., 23(2):153-67, 2001.

Topkara, A. \& Sari, Z. Investigation of third molar impaction in Turkish orthodontic patients: Prevalence, depth and angular positions. Eur. J. Dent., 7(Suppl. 1):S94-8, 2013.

Tortorici, S.; Amodio, E.; Massenti, M. F.; Buzzanca, M. L.; Burruano, F. \& Vitale, F. Prevalence and distribution of odontogenic cysts in Sicily: 1986-2005. J. Oral Sci., 50(1):15-8, 2008.

Varkhede, A.; Tupkari, J. V. \& Sardar, M. Odontogenic tumors: a study of 120 cases in an Indian teaching hospital. Med. Oral Patol. Oral Cir. Bucal, 16(7):e895-9, 2011.

Visscher, C. M.; Lobbezoo, F. \& Schuller, A. A. Dental status and oral health-related quality of life. A population-based study. J. Oral Rehabil., 41(6):416-22, 2014.

World Health Organization (WHO). Oral Health. Oral health information systems. Geneva, World Health Organization (WHO), 2018. Disponible en: www.who.int/oral_health/action/information/ surveillance

Dirección para correspondencia:

Silvia Barrientos Sánchez

Docente /Investigador

Centro de Investigaciones Odontológicas ClO ext. 2899

Pontifica Universidad Javeriana

COLOMBIA

Email: barrien@javeriana.edu.co

Recibido : 02-08-2017

Aceptado: 08-01-2018 